\title{
Approaches to adaptive control of smoke removal rooflight based on mathematical modelling
}

\author{
Aleksandr V. Pilipenko*, Daniil A. Zolotukhin, Anastasia Y. Pilipenko \\ Orel State University, 302026 Orel, Russia
}

\begin{abstract}
In this paper, the authors analyze the design features of smoke removal zenith light, make calculations not only for the dynamic nature of dome's opening/closing, but also taking into account all the forces affecting the object of investigation. Based on the suggested analysis, the authors develop a mathematical model that is proposed to be used for the purpose of developing a corrective effect in the algorithms of the system, which allows judging the adaptability.
\end{abstract}

\section{Introduction}

Zenith lights for Russia are a new phenomenon, despite the fact that in Europe they have been used for more than half a century. Practice of operation shows that their functional significance is very large when they are used in buildings such as: production complexes, shopping and sports halls, winter gardens, flat roofs of a large extent. Zenith lights can be used to organize natural ventilation, but still their main purpose is to provide a natural outlet of smoke through the roof in case of fire or smoke, to prevent the death of people from suffocation, to increase the time of safe evacuation, to preserve sufficient visibility to save people, to reduce the destructive effect of fire and smoke on property and equipment.

The use of zenith lights and smoke removal hatches in Russia depends on the region in which the device will be used, most of the proposed zenith lights can only cope with the IIIrd snow area. In a number of cases this is due to a weak electro/hydro/pneumatic actuator or weak used materials, which are not intended for heavy loads. Due to the large snow loads, the use of smoke removal hatches is not possible, therefore in Russia conventional zenith lights are used. It also explains the so popular use of these devices in European countries, where the snow region does not exceed the II-nd level of the load.

In this work, we set the task to combine a zenith light and a smoke removal light with a subsequent introduction of an automated control system that adapts to climatic and situational conditions. The developed control system of the zenith light presupposes complete automation of operation processes, which was not previously realized. Figure 1 is a block diagram of a control system consisting of a weather module that includes a wind sensor and a rain sensor, a room temperature module, a smoke concentration sensor in the room, and a fire alarm system. The system uses the weather module to determine the speed

* Corresponding author: $\underline{\mathrm{a} @ \text { pilipenko.info }}$ 
and direction of the wind, determines the rain intensity using the rain sensor and, depending on these parameters, automatically determines to what angle it can be opened/closed. The temperature module determines the room temperature and if the temperature has fallen below the set value, the hatch cover closes at a certain angle and vice versa. Using the smoke concentration sensor, the system opens the hatch cover automatically at an angle corresponding to the smoke saturation in the room. All occurs in an automatic mode based on comparing operation parameters of real system and calculated mathematical models in the control block which determines an angle of opening/closing of a hatch cover.

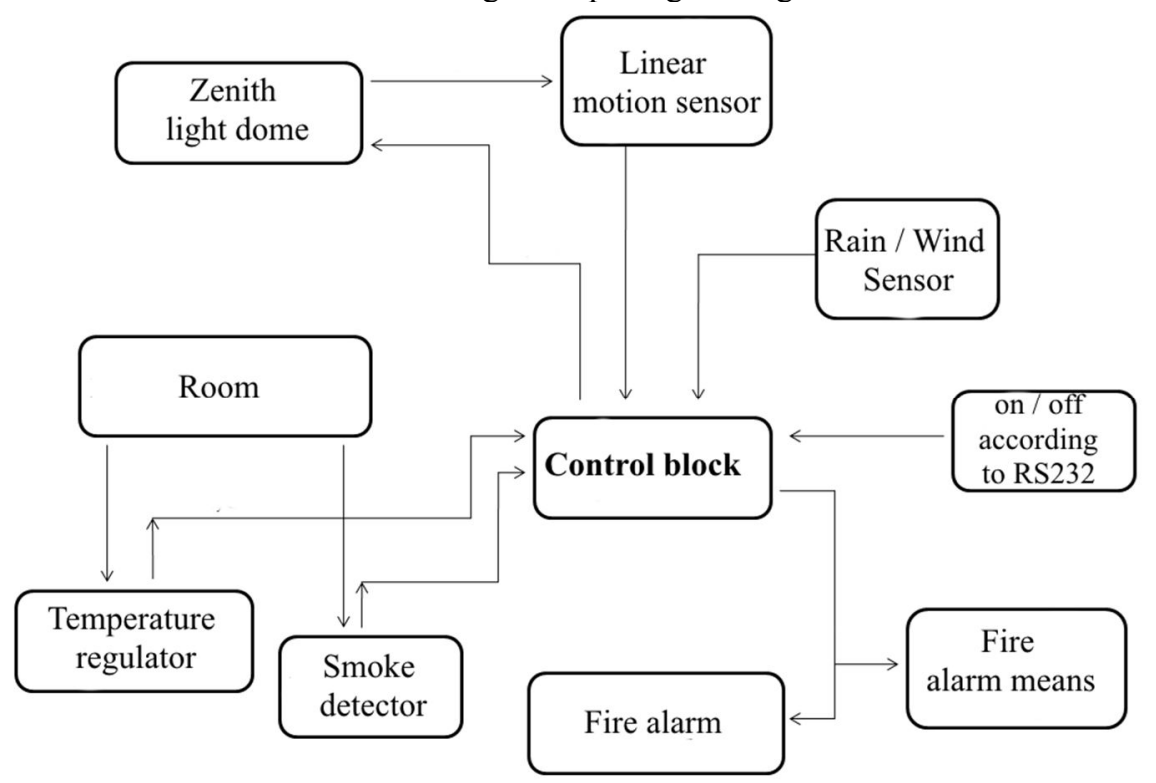

Fig. 1. Structural diagram of the control system.

\section{Modeling}

Based on the foreign patent EP20110188468, its design part, the kinematic scheme and the fixing details of the dome base, adapted for our conditions, were redeveloped. The existing smoke dome is not suitable for heavy loads in difficult weather conditions. Often, the problem is that a strong wind or heavy snow prevents the normal functioning of the entire system. Some nodes, for example, such as an electric drive simply cannot cope with the lifting of the hatch cover in case of snow load. In order to adapt the smoke removal light for our conditions, it is necessary to strengthen the design of the lever system and put a more powerful electric motor. In addition to snow loads, the wind load system affects the smoke removal system, when wind gusts reach $40 \mathrm{~m} / \mathrm{s}$. These wind loads can cause damage to the fastening cover, damage to the electric drive, as well as failure of the entire system as a whole.

Long-term modelling and strength calculations in APM and SolidWorks systems resulted in the design of the drive (Figure 2), which had welded levers that were made of sheet steel of different thickness. When designing and creating a new construction, an important thing was that the opening angle remained more than $140^{\circ}$. This task was successfully accomplished due to the unique electric drive system "Actulux"[1]. 


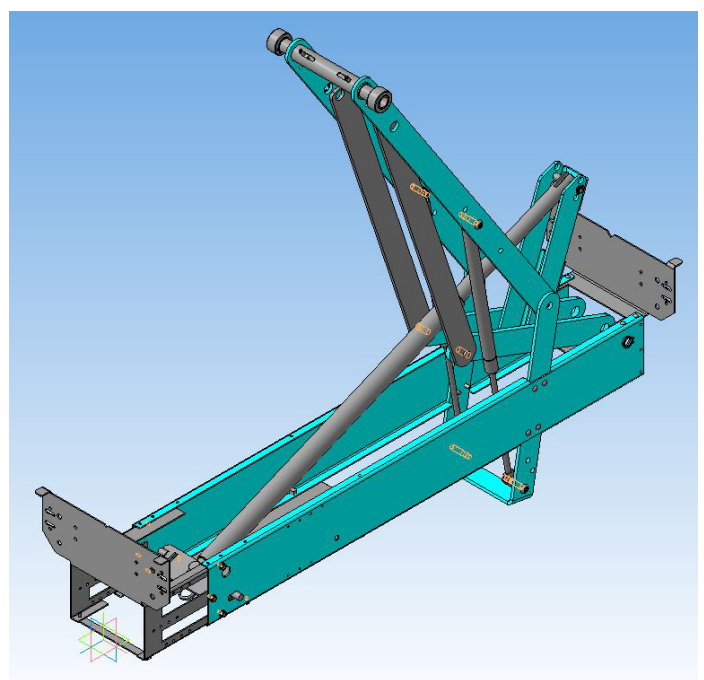

Fig. 2. Design of the dome control mechanism.

The mathematical model of the dome opening/closing mechanism was created on the basis of the kinematic model, which is a relation between the linear extension of the actuator rod and the opening angle of the zenith lamp (Figure 3). Under the pushing effect, the motor drives the rod 12 to the position determined by the value $\mathrm{S}-$ the stem extension. With the force of the rod on the ACB, the latter starts to rotate around the fixed axis B, which subsequently drives the ED slider fixed to the D axis and, finally, lifts the dome of the zenith light EF fixed to the axis F.

The original length data is taken from the detail drawings:

$11=115 ; 12=400 ; 13=205 ; 14=153 ; 15=153 ; 16=540 ; 17=545 ; 18=110 ; 19=940 ; 110=605$;

$\mathrm{S} 1=670 ; \mathrm{S} 2=588 ; \mathrm{S} 3=905 ; \mathrm{H} 1=50 ; \mathrm{H} 2=272 ; \mathrm{H} 3=172$;

$\mathrm{xf}=\mathrm{S} 3 ; \mathrm{yf}=\mathrm{H} 3 ; \mathrm{xg}=\mathrm{xe} ; \mathrm{yg}=\mathrm{yf}$;

$\mathrm{S}=100 ;$ alfa $=52$.

To make up the equations of motion of each link, it is necessary to determine the coordinates of each moving point: $\mathrm{A}, \mathrm{C}$ and $\mathrm{E}$. We write the equations of motion for each point in the form of equation (1).

$$
\left\{\begin{array}{c}
x a^{2}+y a^{2}=(l 1+l 2+S)^{2} \\
(x a-S 1)^{2}+(y a-H 1)^{2}=(l 3 \cdot \sin \alpha)^{2}
\end{array}\right.
$$

From the created system of equations, we find the coordinates $X a, Y a(2)$ and (3).

$$
\begin{gathered}
x a=\sqrt{(l 1+l 2+S)^{2}-y a^{2}}, \\
y a=\frac{H 1 \cdot\left((l 1+l 2+S)^{2}+S 1^{2}+H 1^{2}+(l 3 \cdot \sin \alpha)^{2}\right)}{2 \cdot\left(H 1^{2}+S 1^{2}\right)},
\end{gathered}
$$




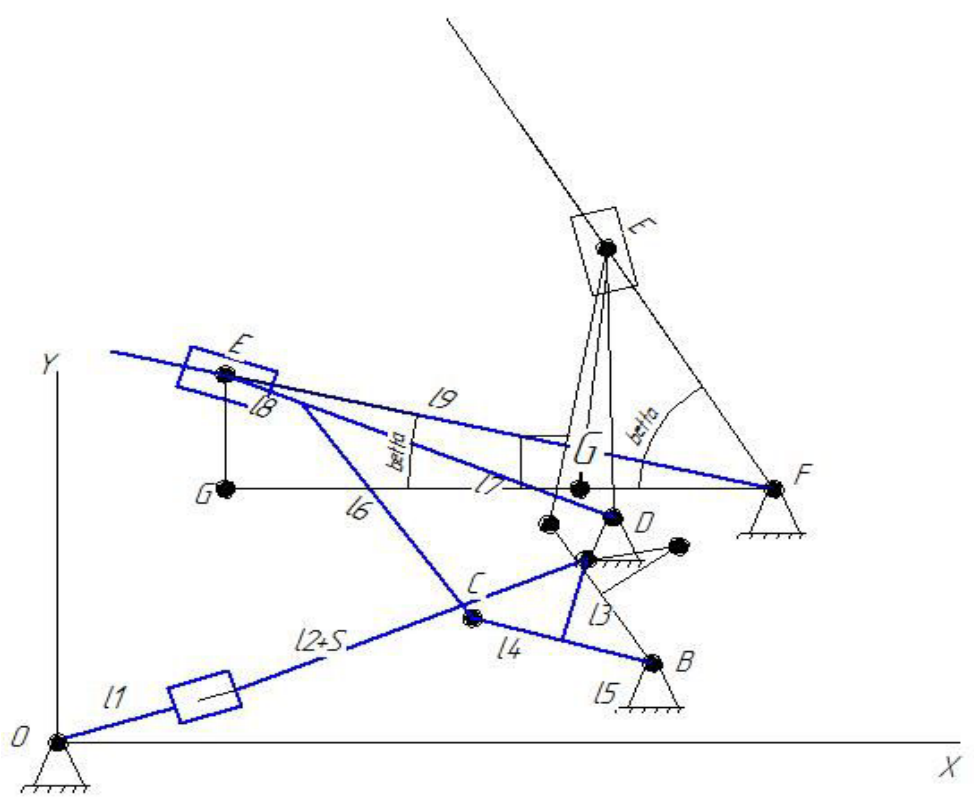

Fig.3. Kinematic model of the mechanism.

After finding the point $\mathrm{A}$, we find the equation of motion for point $\mathrm{C}$ and write it in the form of equation (4).

$$
\left\{\begin{array}{c}
(x c-S 1)^{2}+(y c-H 1)^{2}=(l 4 \cdot 2)^{2} \\
(x c-x a)^{2}+(y c-y a)^{2}=(l 3 \cdot \sin \alpha)^{2},
\end{array}\right.
$$

Using (4), we find the coordinates of the point $\mathrm{C}-X c$ and $Y_{c}$ according to formulae (5) and (6).

$$
\begin{gathered}
x c=\sqrt{(l 4 \cdot 2)^{2}-(y c-H 1)^{2}}+S 1, \\
y c=\frac{-\left(8 \cdot(S 1-x a)^{2} \cdot H 1 \cdot 4 \cdot(H 1-y a) \cdot\left((l 3 \cdot \sin \alpha)^{2}-(l 4 \cdot 2)^{2}+H 1^{2}-(S 1+x a)^{2}-y a\right)\right)}{-8 \cdot(S 1-x a)^{2}-8 \cdot(H 1-y a)^{2}},
\end{gathered}
$$

Then we find the coordinates of the slider, for the point E, using equation (7):

$$
\left\{\begin{array}{c}
(x \mathrm{e}-S 2)^{2}+(y \mathrm{e}-H 2)^{2}=(l 7+l 8)^{2} \\
(x e-x c)^{2}+(y e-y c)^{2}=l 10^{2}
\end{array}\right.
$$

Based on (7) we find the coordinates of the point E according to formulae (8) and (9).

$$
\begin{gathered}
x e=\sqrt{(l 7+l 8)^{2}-(y e-H 2)^{2}}+S 2 \\
y c=\frac{-\left(8 \cdot(S 2-x c)^{2} \cdot H 2 \cdot 4 \cdot(H 2-y c) \cdot\left(l 10^{2}-(l 7+l 8)^{2}+H 2^{2}-(S 2+x c)^{2}-y c\right)\right)}{-8 \cdot(S 2-x c)^{2}-8 \cdot(H 2-y c)^{2}}
\end{gathered}
$$

Knowing the coordinates of all points, we find the relationship for calculating the opening angle of the hatch cover and the linear displacement of the rod by formula:

$$
\sin \beta=\frac{\sqrt{(x f-x e)^{2}+(y e-y f)^{2}}}{\sqrt{(y e-y g)^{2}}}
$$


Figure 4 shows how the opening angle of the zenith cover changes from the movement of the motor rod after establishing the relationship.

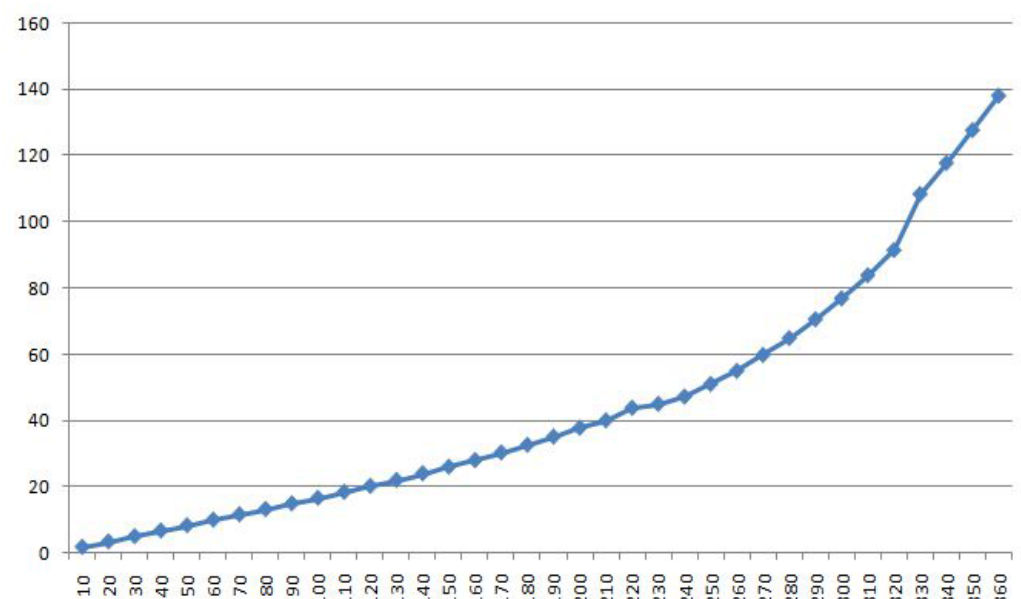

Fig.4. Graph of the opening angle versus the movement of the motor rod.

\section{Algorithmization}

The development of algorithms considering the obtained models will allow the automated system to determine the angle of the dome lifting and take into account the linear value to which the rod will extend $[2,3]$. The developed algorithms in the form of a block diagram are shown in Figure 5, where: 1 - Start of the program; 2 - data collection from sensors and industrial interface RS232, which provides remote control and control of emergency systems; 3 - selection of the operating mode of the system between training 4 and normal operation $5 ; 6$ - calculation of the mathematical model; 7 - subprogramme for comparing data from sensors and simulation results; 8 - subprogramme of controlling action development to approximate the system operation parameters to simulation results; 9 - if the controlling action is produced, the data is formed into a table with time and sent to the database; 13 - sending of the controlling impact on the actuating mechanisms; 14 selection of the controlling action for correcting the operation of the system to match the model (comparing the solutions developed with the allowable corrective coefficients); $15,16,17,18,19$ - correcting effects and operation parameters of the automated system; 20 if the self-study mode is selected, then the selection of correction factors for the mathematical model to bring it to fit to the real system occurs; 26 - sending effects and collecting data from sensors; 27, 28 - conversion and processing of data from sensors; 29 a request to the subprogramme for comparing data from sensors and simulation results; 30 - sending of the controlling action to the actuators; 31 - a request to the subprogramme correction of the mathematical model to introduce correction factors into it. 32 - check whether the training mode is still on; 33 - whether the selected operation parameters have been fulfilled; 34 - check of supply availability; 35 - the end. 


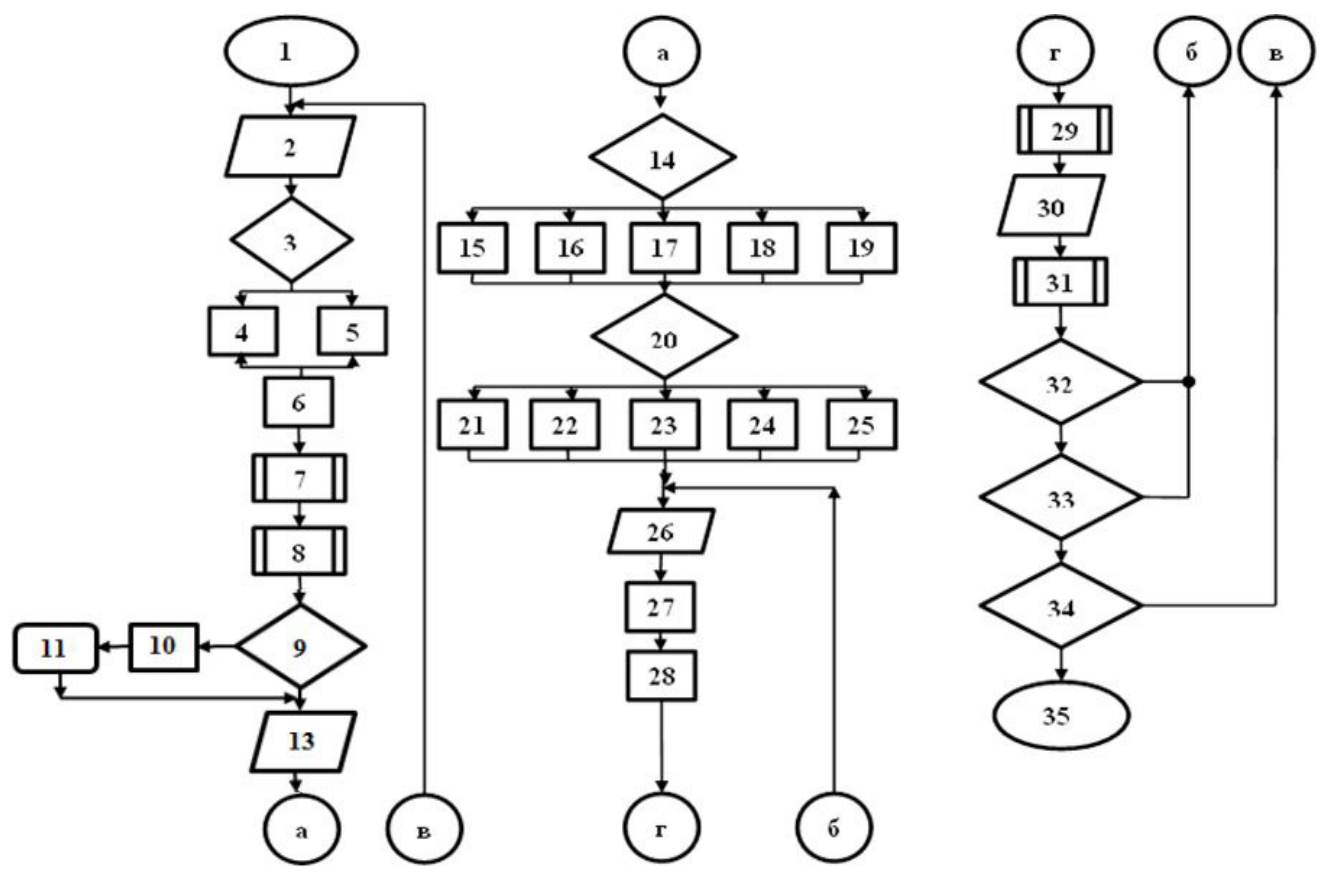

Fig.4. Graph of the opening angle versus the movement of the motor rod.

\section{Conclusions}

As it can be seen from the presented algorithm, such a system is able not only to scale itself, by controlling from other devices via RS 232, but is also able to operate adaptively in two modes, either adapting the mathematical model to real climatic conditions of operation, or adapting the system operation to a model. Such operating modes allow not only using zenith lights in completely different conditions, but also increasing the energy efficiency of building control systems by determining the exact opening angle for the required ventilation parameters of the room.

\section{References}

1. D.A. Zolotukhin, Vibration technologies, mechatronics and controlled machines: Sat. sci. article: at 2 am Part 2 / rare: SF Yatsun (Edited by Ed.) [And others] (SouthWestern State University, Kursk, 424 (2014)

2. D.A. Zolotukhin, NI NIDays-2014: Proceedings of the XIII International Scientific and Practical Conference, 460, 2014

3. A.V. Pilipenko, S.P. Petrov, MATEC Web Conf, 155, 01041 (2018) 